\title{
Understanding the Role of Oxidative Etching in the Polyol Synthesis of Pd Nanoparticles with Uniform Shape and Size
}

\author{
Yujie Xiong, ${ }^{\dagger}$ Jingyi Chen, ${ }^{\dagger}$ Benjamin Wiley, ${ }^{\dagger}$ and Younan Xia ${ }^{*},{ }^{\dagger}$ \\ ${ }^{\dagger}$ Department of Chemistry \\ ${ }^{\star}$ Department of Chemical Engineering \\ University of Washington, Seattle, Washington 98195-1700 \\ *Corresponding author.E-mail: xia@chem.washington.edu
}

Shaul Aloni and Yadong Yin

The Molecular Foundry

Lawrence Berkeley National Laboratory, Berkeley, California 94720 


\section{Experimental Procedure:}

In a typical synthesis, $5 \mathrm{~mL}$ of ethylene glycol (EG, J. T. Baker, 9300-01) was placed in a 3-neck flask (equipped with a reflux condenser and a magnetic Teflon-coated stirring bar) and heated in air at $110{ }^{\circ} \mathrm{C}$ for $1 \mathrm{~h}$. Meanwhile, $0.1384 \mathrm{~g}$ of palladium(II) sodium chloride $\left(\mathrm{Na}_{2} \mathrm{PdCl}_{4}, \mathrm{Aldrich}\right.$, 379808-1g) and $0.0800 \mathrm{~g}$ of poly(vinyl pyrrolidone) (PVP, M.W.=55,000, Aldrich, 856568-100g) were separately dissolved in $3 \mathrm{~mL}$ of EG at room temperature. These two solutions (with the molar ratio between $\mathrm{Pd}$ and the repeating unit of PVP being 2:3) were then injected simultaneously into the flask through a syringe pump at a rate of $45 \mathrm{~mL}$ per hour. Heating of the reaction at $110^{\circ} \mathrm{C}$ was continued in air for $9 \mathrm{~h}$. A set of samples were taken over the course of each synthesis with a glass pipet. To minimize temperature perturbation during sampling, the glass pipet was held just above the solution and preheated for $30 \mathrm{~s}$ before immersion. For argon-

protected synthesis, the other experimental parameters were kept the same except for the continuous bubbling of argon. The samples were washed with acetone and then with ethanol several times to remove most of the EG and PVP by centrifugation. The as-obtained samples were then characterized by transmission electron microscopy (TEM), high-resolution TEM (HRTEM), dark-field TEM, electron diffraction (ED), and powder X-ray diffraction (PXRD).

\section{Instrumentation:}

A drop of the aqueous suspension of particles was placed on a piece of carbon-coated copper grid (Ted Pella, Redding, CA, for TEM) and dried under ambient conditions. The grid was then transferred to a gravity-fed flow cell and washed for $1 \mathrm{~h}$ with deionized water to remove the excess PVP. Finally the sample was dried and stored in vacuum for TEM characterization. TEM images and ED patterns were taken on a Phillips 420 transmission electron microscope operated at $120 \mathrm{kV}$. HRTEM and dark-field TEM images were taken on a Jeol 2010 LaB6 high-resolution transmission electron microscope operated at $200 \mathrm{kV}$. PXRD patterns were recorded on a Philips 1820 diffractometer equipped with a $\mathrm{Cu}-\mathrm{K} \alpha$ radiation source $(\lambda=1.54180 \AA)$. UV-vis spectra were obtained using a Hewlett Packard 8452A diode array spectrophotometer. 

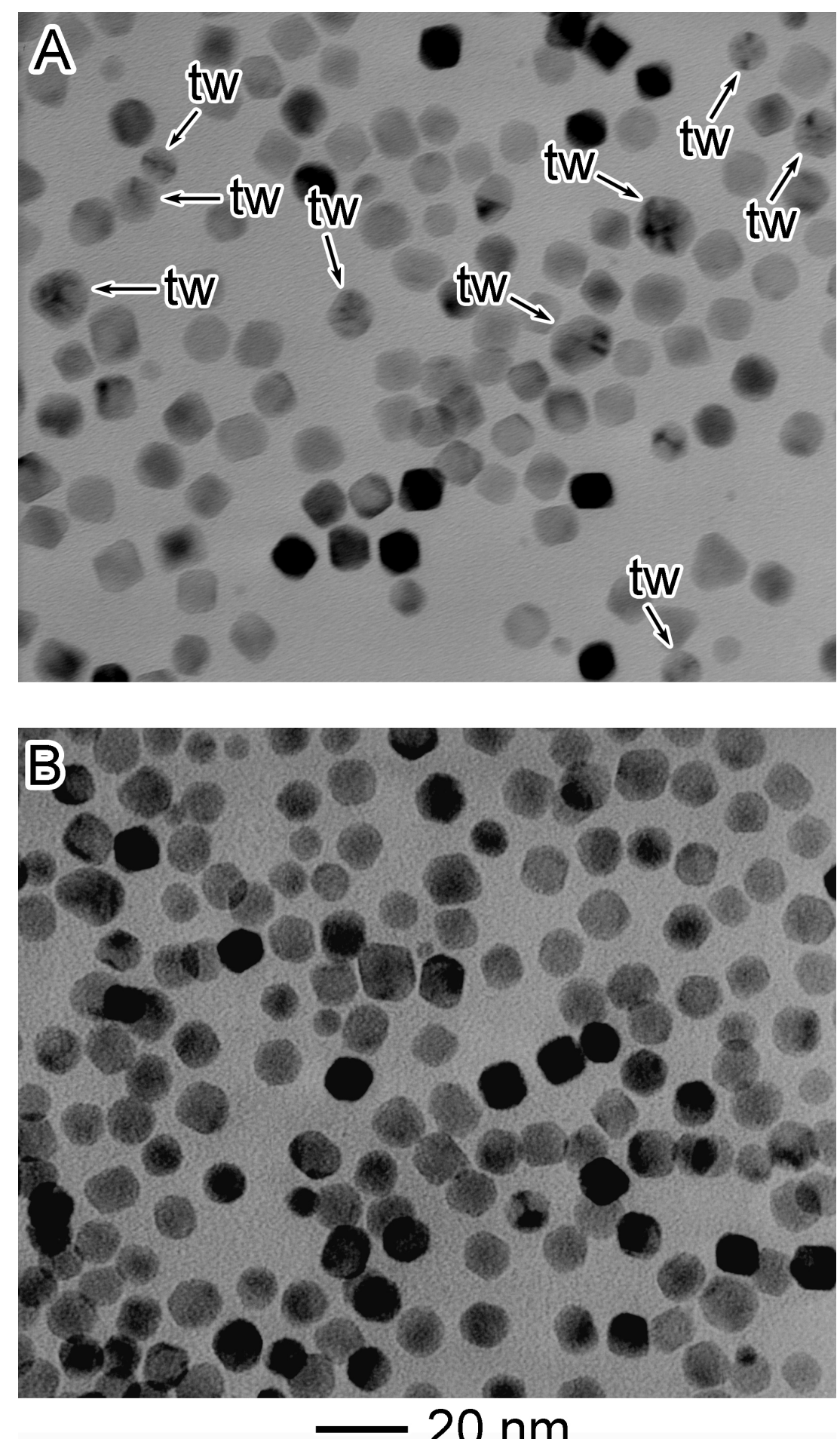

Figure S1. TEM image of the sample prepared in air at (A) $t=5$ min and (B) $t=3 \mathrm{~h}$. At $t=5$ min, the sample mainly contained cubooctahedra of 4-8 $\mathrm{nm}$ in size and $10 \%$ twinned particles (labeled by $t w$ ). As the reaction proceeded to $\mathrm{t}=1 \mathrm{~h}$, all the twinned particles disappeared while the average size of the cubooctahedra grew to $8 \mathrm{~nm}$. 

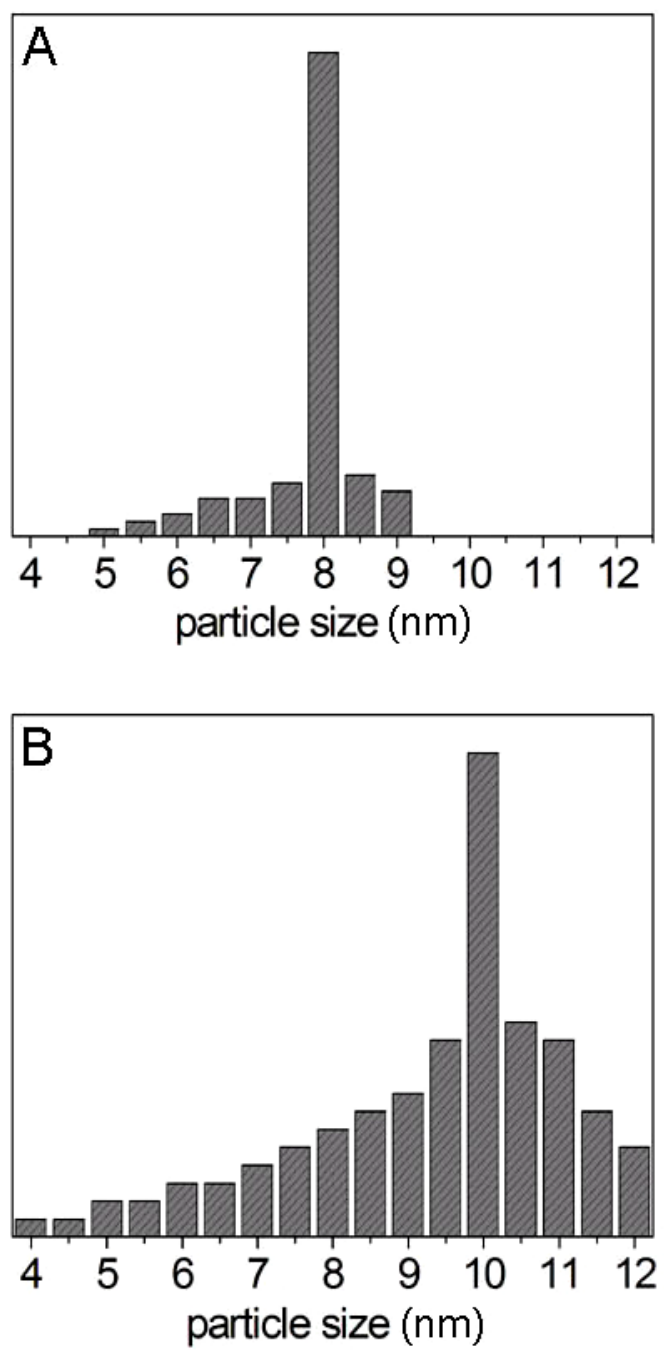

Figure S2. Plot of size distribution for the sample collected at (A) $t=3 \mathrm{~h}$ and (B) $t=7 \mathrm{~h} 40 \mathrm{~min}$, respectively. Based on the analysis of 100 particles on TEM images, the particles at $\mathrm{t}=7 \mathrm{~h} 40 \mathrm{~min}$ (B) had a much broader size distribution than that at $t=3 \mathrm{~h}$ (A). 

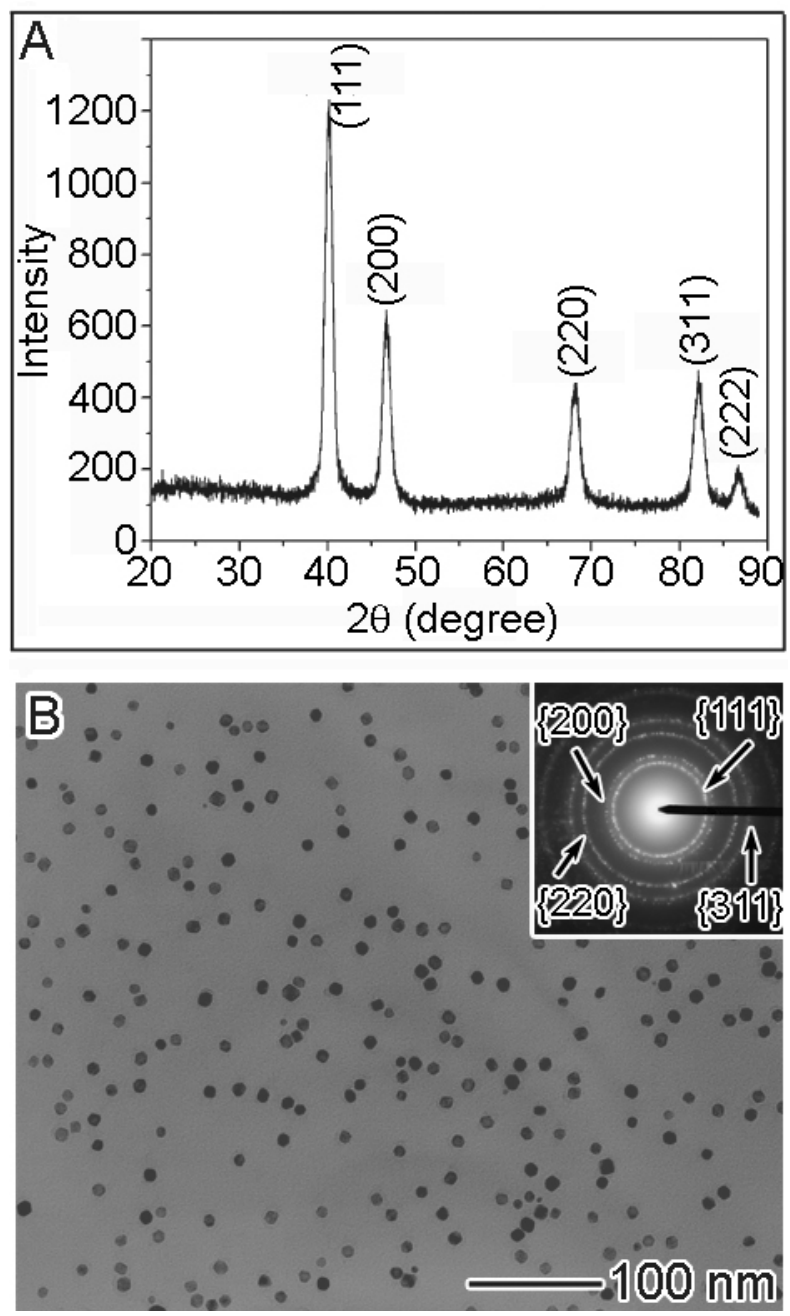

Figure S3. (A) PXRD pattern taken from Pd cubooctahedral nanoparticles prepared in air at $t=3$ h. (B) TEM image and ED pattern of another different batch of Pd cubooctahedral nanoparticles prepared in air at $\mathrm{t}=3 \mathrm{~h}$. In the XRD pattern, all the peaks can be indexed to face-centered cubic (fcc) palladium (JCPDS card, 05-0681). The ED pattern (inset) taken from an assembly of Pd cubooctahedral nanoparticles, indcating that they are highly crystalline. The rings can be indexed to the diffraction from $\{111\},\{200\},\{220\}$ and $\{311\}$ planes of $f c c$ palladium, respectively. 

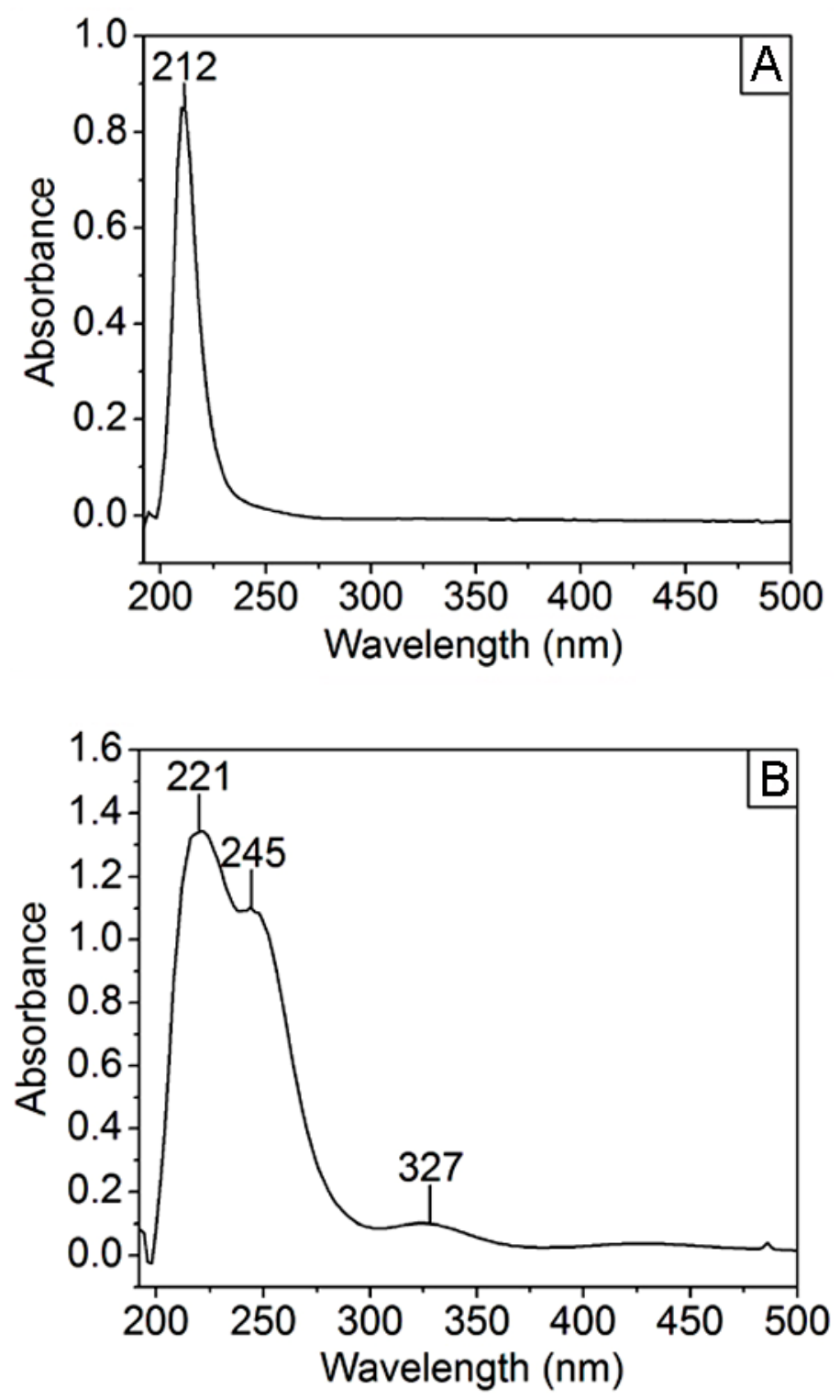

Figure S4. (A) UV-vis spectrum of PVP in ethylene glycol. (B) UV-vis spectrum of $\mathrm{Na}_{2} \mathrm{PdCl}_{4}$ dissolved in ethylene glycol. These UV-vis spectra indicate that the absorption peak at $212 \mathrm{~nm}$ can be attributed to PVP and the peaks at 221, 245, and $327 \mathrm{~nm}$ to $\left[\mathrm{PdCl}_{4}\right]^{2-}$. These assignments are consistent with the literature (Veisz, B.; Király, Z. Langmuir 2003, 19, 4817; Chen, J.; Herricks, T.; Geissler, M.; Xia, Y. J. Am. Chem. Soc. 2004, 126, 10854). 

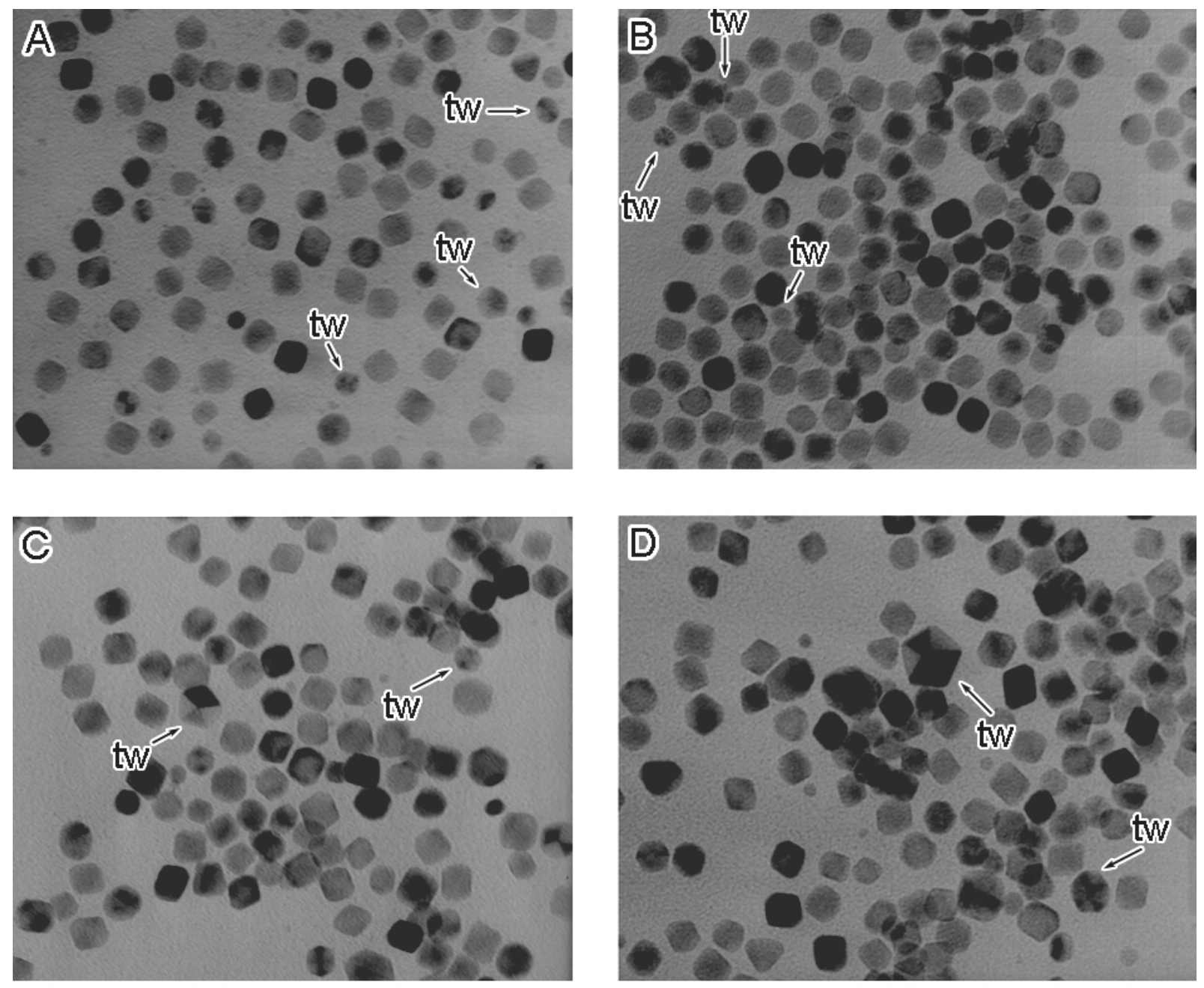

$20 \mathrm{~nm}$

Figure S5. TEM images of $\mathrm{Pd}$ nanoparticles synthesized under argon at (A) $t=30 \mathrm{~min}$; (B) $\mathrm{t}=3 \mathrm{~h}$; (C) $\mathrm{t}=6 \mathrm{~h}$; and (D) $\mathrm{t}=9 \mathrm{~h}$. Twinned particles are labeled by $t w$. The $20-\mathrm{nm}$ scale bar is applicable to all images. When the reaction was performed under argon, the twinned nanoparticles remained in all the products and the single-crystal cubooctahedra were not dissolved as the reaction time was prolonged. These observations also confirm that oxidative etching by air was responsible for the dissolution of twinned particles and the decrease in size for the cubooctahedral nanoparticles. 

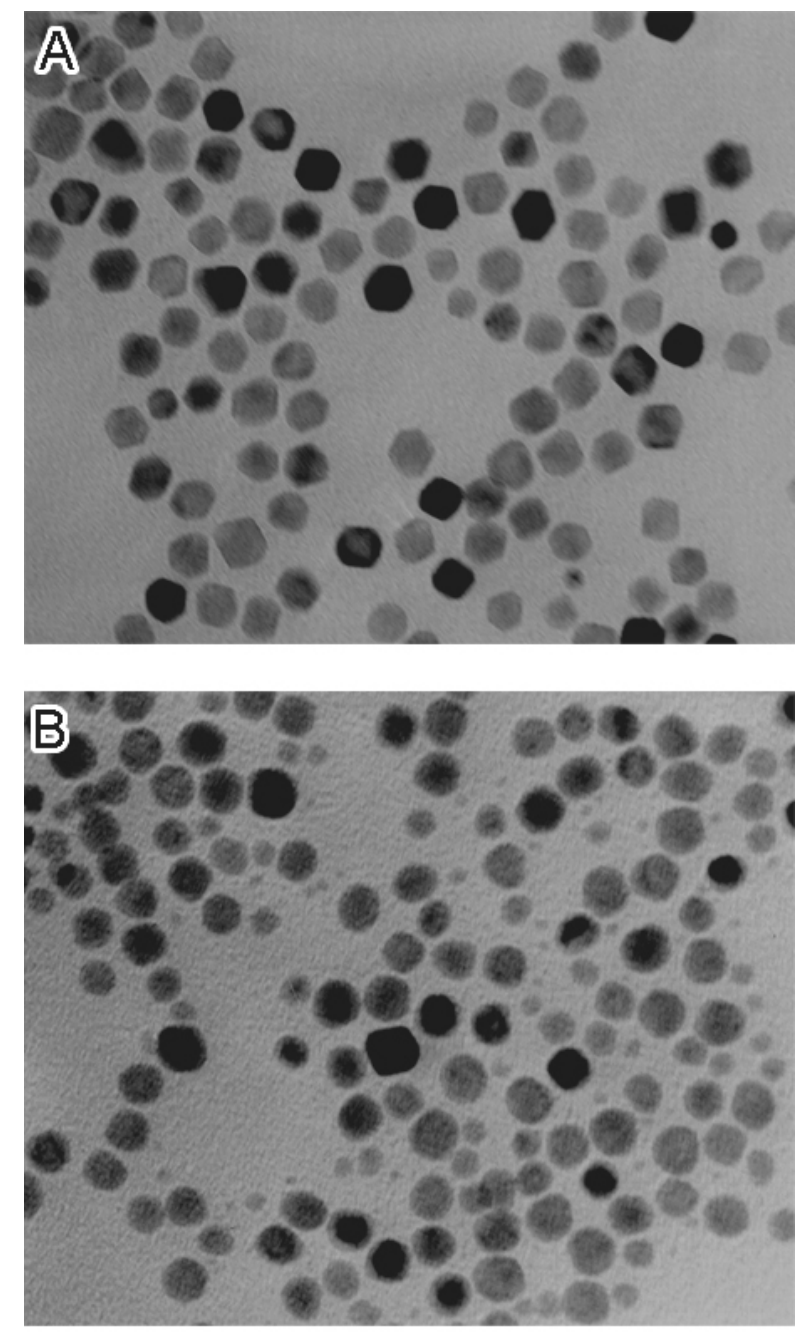

$20 \mathrm{~nm}$

Figure S6. TEM image of the sample prepared under argon at $t=3 \mathrm{~h}$ after the solution was exposed to air at room temperature for (A) $12 \mathrm{~h}$ and (B) 4 days. Before exposure to air, the sample contained $5 \%$ twinned particles (see Fig. S5B). After exposure to air for $12 \mathrm{~h}$, one can see that the twinned particles had disappeared, indicating that they were removed due to oxidative etching. After a longer period of exposure, the single-crystal, cubooctahedral nanoparticles were also partially dissolved. To obtain single-crystal Pd nanoparticles with uniform shape and size, one needs to stop etching at the right stage. 


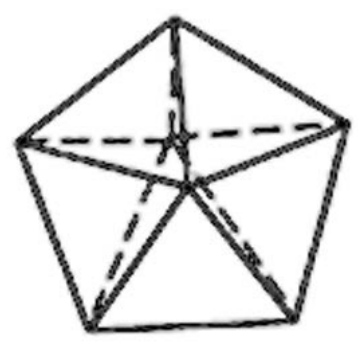

Decahedron

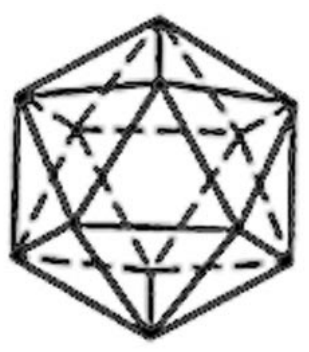

Icosahedron

Figure S7. Geometrical shapes of multiply twinned decahedral and icosahedral particles. Generally, the 5-fold twinned, decahedral particle is described as a mosaic structure consisting of five single crystal tetrahedra oriented radially about a central axis so that all five tetrahedra share a common edge in the center, and each tetrahedron has two sides in contact with its neighbors. However, this model is not a space filling one, since each tetrahedron can share an angle of only $70.5^{\circ}$, leaving a $7.5^{\circ}$ gap. To make up for this difference, significant lattice distortions, surface relaxations, and defects must occur, and these have been observed experimentally in a number of high-resolution TEM studies of twinned nanoparticles (Wang, Z. L. J. Phys. Chem. B 2000, 104, 1153; Nepijko, S. A.; Levlev, D. N.; Schulze, W.; Urban, J.; Ertl, G. ChemPhysChem 2000, 1, 140). The necessarily higher density of defects on their surfaces results in the higher reactivity of twinned Pd particles. 